\title{
Continuing U.S. Participation in the LHC Accelerator Program 1
}

\author{
M. J. Syphers \\ Fermilab, P.O. Box 500, Batavia, IL 60510, USA
}

\begin{abstract}
The U.S. LHC Accelerator Research Program (LARP) was established to enable U.S. accelerator specialists to take on active and important roles in the LHC accelerator project during its commissioning and early operations, and to be a major collaborator in future LHC performance upgrades. It is hoped that this follow-on effort to the U.S. contributions to the LHC accelerator project will improve the capabilities of the U.S. accelerator community in accelerator science and technology in order to more effectively use, develop, and preserve unique U.S. resources and capabilities during the LHC era .
\end{abstract}

Keywords: LHC, LARP, hadron collider

PACS: 29.20.-c, 29.20.Dh, 29.20.Lq

Major U.S. contributions to the Large Hadron Collider project at CERN have been provided under the U.S. LHC Accelerator Project (USLHC) of the Department of Energy. This multi-laboratory project, carried out by Brookhaven National Laboratory, Fermi National Accelerator Laboratory, and Lawrence Berkeley National Laboratory, has developed, built, and delivered electromagnetic components and associated equipment for the LHC. In particular, final-focus triplet quadrupole magnets, beam separation dipole magnets, and interfacing hardware have been built and shipped to CERN for use in the long straight sections of the LHC. [1].

During the final years of the USLHC project, a proposal from the three laboratories was put forth for a continuing presence in the LHC program. The U.S. LHC Accelerator Research Program (LARP) would allow US accelerator specialists to continue to take an active and important role in the LHC accelerator program during its commissioning and operations, and to be a major collaborator in LHC performance upgrades.[2] The program was formally approved in 2003, and after two years of start-up funding has recently reached a level of support allowing major collaborative efforts to take place. In addition, Stanford Linear Accelerator Center has joined the collaboration as well.

Tasks within LARP include efforts to develop new, innovative technologies for, and designs of accelerator magnets to be used in the harsh environment of the LHC interaction regions at luminosities eventually upgraded to $10^{35} \mathrm{~cm}^{-2} \mathrm{~s}^{-1}$ or beyond. They also provide beam instrumentation and beam collimation systems development (for both present and future operations), LHC hardware and beam commissioning expertise, and accelerator physics calculations in support of all of the above.

${ }^{1}$ Work supported by the U.S. Department of Energy under contact No. DE-AC02-76CH03000. 




FIGURE 1. "Fit-up" of one side of triplet magnet assembly at CERN Hall 181.

\section{AREAS OF PARTICIPATION}

With the delivery of the final U.S.-produced components in late 2005, the U.S. LHC Accelerator Project is essentially complete. Through LARP, collaborators were able to participate in a complete "fit-up" of magnet and cryogenics hardware, making up one side of one interaction region, performed in Hall 181 at CERN, directly above the CMS detector region. (See Figure 1.) Installation of this hardware in the tunnel is already taking place, with LARP personnel participating in the process as well as in its initial commissioning.

Meanwhile, in late 2004 a report was issued from CERN citing a need for more manpower to assist in the commissioning of cryogenic and electrical hardware in order to keep to the LHC installation and commissioning schedule.[3] Of the 40 or so "missing staff" outlined in the report, LARP is gearing up to send highly specialized personnel from the U.S. labs to assist with magnet, power supply, and cryogenic systems over the next year. Additionally, it is anticipated that several accelerator physicists from LARP will be participating in beam commissioning at CERN.

In addition to commissioning LHC Project hardware and beam, several LARP collaborators are working on additional beam instrumentation devices which were not included in the original LHC project scope. These include luminosity monitors for the two largedetector interaction regions, a tune feedback system for continuous measurement and control of betatron oscillation frequencies, and a Schottky monitor system for measuring tune distributions and chromaticity.

\section{Luminosity Upgrades}

Beyond the immediate tasks of installing and commissioning hardware and establishing circulating beam in the LHC, LARP is also participating - and in some cases, taking the lead - in investigations of future luminosity upgrades to the collider. Upgrades to higher luminosity require radiation-hard strong-focusing triplet magnets, and upgraded collimators in the arcs to deal with the higher beam intensity. The power emanating 

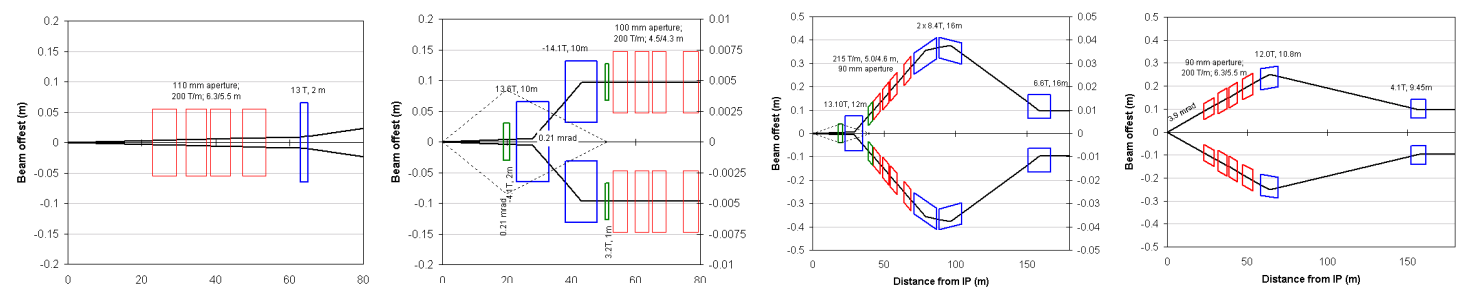

FIGURE 2. Possible LHC IR upgrades being investigated by LARP, showing different configurations in plan view of one-half of an IR. The interaction point is at the left.

from the interaction point toward the $1.8^{\circ} \mathrm{K}$ superconducting magnets at a luminosity of $10^{34} \mathrm{~cm}^{-2} \mathrm{sec}^{-1}$ is approximately $700 \mathrm{~W}$ in each direction, $7 \mathrm{~kW}$ for an upgraded IR (compared to $\sim 1 \mathrm{~W}$ in the Tevatron). The stored energy in each beam is almost $400 \mathrm{MJ}$, and even higher for an upgrade (compared to $\sim 1 \mathrm{MJ}$ in the Tevatron). While LHC installation has not yet finished, now is the time to begin R\&D on hardware development for future upgrades. The LARP magnet program is investigating issues such as magnet design concepts, cryogenic heat transfer issues, radiation heat deposition, and conductor and cable development. Possible upgrades to the LHC collimation and machine protection system are being looked at, including tertiary collimators for the arcs of the collider. Various possible modifications to the optics and layout of the high luminosity interaction regions are being studied (see Figure 2) in conjunction with the magnet work. Additional accelerator physics support is investigating electron cloud issues at higher beam currents as well as beam-beam interaction effects and possible corrections at higher luminosities.

\section{OUTLOOK}

The evolution of LARP over the past three years has seen the program become a structured entity, with work breakdown structures, change control, committees and DOE reviews. With roughly 100 participants from 4 national laboratories, LARP personnel are already appearing at CERN and luminosity monitors and other beam instrumentation is on schedule for delivery in time for LHC start-up. Magnet and beam collimation efforts are progressing toward future upgrades for even higher luminosity, exploring various IR layouts and options, with associated accelerator physics efforts in support. The program is also providing short-term appointments (Toohig Fellowships) to recent PhD's to work on LHC-related R\&D.[5]

\section{REFERENCES}

1. Details and further information available at http://wWw-td. fnal . gov/LHC/USLHC.html.

2. "The U.S. LHC Accelerator Research Program: A Proposal," available at http://www . rhichome.bnl.gov/LARP/papers/LARP_Proposal.pdf

3. "Resources for Hardware Commissioning," LHC Proj. Doc. LHC-PM-MR-0002 (2004).

4. Further information available at http: //uslarp.org.

5. See http://www.interactions.org/toohig/for more information. 